\title{
Sensitivity analysis of large-eddy simulations to subgrid-scale-model parametric uncertainty using polynomial chaos
}

\author{
DIDIER LUCOR ${ }^{1}$, JOHAN MEYERS ${ }^{1,2}$ \\ AND PIERRE SAGAU T \\ ${ }^{1}$ D'Alembert Institute, Université Pierre et Marie Curie - Paris 6 \\ Boîte 162, 4 place Jussieu, 75252 Paris cedex 05, France \\ ${ }^{2}$ Department of Mechanical Engineering, Katholieke Universiteit Leuven \\ Celestijnenlaan 300A, B3001 Leuven, Belgium \\ lucor@lmm.jussieu.fr; meyers@lmm.jussieu.fr, johan.meyers@mech.kuleuven.be; \\ sagaut@lmm.jussieu.fr
}

(Received 19 July 2006 and 28 March 2007)

We address the sensitivity of large-eddy simulations (LES) to parametric uncertainty in the subgrid-scale model. More specifically, we investigate the sensitivity of the LES statistical moments of decaying homogeneous isotropic turbulence to the uncertainty in the Smagorinsky model free parameter $C_{s}$ (i.e. the Smagorinsky constant). Our sensitivity methodology relies on the non-intrusive approach of the generalized Polynomial Chaos (gPC) method; the gPC is a spectral non-statistical numerical method well-suited to representing random processes not restricted to Gaussian fields. The analysis is carried out at $R e_{\lambda}=100$ and for different grid resolutions and $C_{s}$ distributions. Numerical predictions are also compared to direct numerical simulation evidence. We have shown that the different turbulent scales of the LES solution respond differently to the variability in $C_{s}$. In particular, the study of the relative turbulent kinetic energy distributions for different $C_{s}$ distributions indicates that small scales are mainly affected by changes in the subgrid-model parametric uncertainty.

\section{Introduction}

Large-eddy simulation (LES) is an often-used computational method to predict turbulent flows in various applications. In order to control the computational cost associated with such predictions, LES reduces the dynamic complexity of the NavierStokes equations by means of a low-pass filter. A side effect is the emergence of subgrid-scale stresses, which have to be modelled by means of a subgrid-scale model.

Subgrid-scale modelling in LES has been the subject of a large number of studies in recent decades (see e.g. Sagaut 2006, for an overview). Regrettably, as reviewed by Jiménez \& Moser (2000), one can estimate, based on conditional averages, that the statistical error between the 'best' model based on resolved properties and the effective subgrid-scale stresses is at best of the order of $80 \%$. This not only illustrates the difficulty in the formulation of subgrid-scale closures, but also shows the limitations of the LES approach to predicting turbulence, indicating that instantaneous LES solutions will quickly diverge from the exact filtered solution. It is however one of the central assumptions in LES that statistically averaged properties, such as mean 
profiles, the average turbulent kinetic energy distribution, etc., can be predicted well when a suitable subgrid closure is used (Meneveau 1994).

In most cases, a deterministic approach has been followed for the formulation of models. That is, typically based on the filtered velocity field $\overline{\boldsymbol{u}}$ and its gradients $\nabla \otimes \overline{\boldsymbol{u}}$, and using physical insight into turbulence, an expression $m_{i j}(\overline{\boldsymbol{u}}, \nabla \otimes \overline{\boldsymbol{u}}, \ldots)$ is proposed which should approximate on average the effect of the effective unclosed subgrid stresses $\tau_{i j}=\bar{u}_{i} u_{j}-\bar{u}_{i} \bar{u}_{j}$. To this end, models should in the first instance yield the correct level of subgrid dissipation, while, e.g. in boundary layers, the distribution of subgrid transport is also very important in determining good simulation results (Völker, Moser \& Venugopal 2002). Further properties which are thought valuable for models are the preservation of symmetry properties of the differential equations, the inclusion of back-scatter effects, the prediction of intermittency levels, realizability, etc. (see among others Vreman, Geurts \& Kuerten 1994; Meneveau 1994; Fureby \& Tabor 1997; Ghosal 1999; Carati, Winckelmans \& Jeanmart 2001, for more information).

In simulations, subgrid-scale models do not always yield their theoretically expected solution, and this is often shown in the significant discrepancy between a priori tests and $a$ posteriori results. This is caused by simplifications and assumptions related to the shape of the LES filter, the type of turbulence at the filter cutoff, etc., in combination with the nonlinear nature of the Navier-Stokes equations. All this leads to a level of uncertainty in the performance of subgrid-scale models and the quality of simulation results, most notoriously when the models contain an adaptable model parameter, such as the well-known Smagorinsky (1963) model.

In Smagorinsky LES, the correct selection of the model parameter $C_{s}$ is central for the quality of results. In early literature, $C_{s}$ was often hypothesized to be a universal constant related to the model. Different values for $C_{s}$ are quoted, such as 0.1 (Deardoff 1970), 0.15 (Pope 2000), 0.17 (McMillan \& Ferziger 1979), 0.17 (Lilly 1967), 0.185, and 0.23 (Lilly 1966). Some of these values are a result of an empirical fit, while others come from theoretical estimations. More recently, it was shown that the Smagorinsky coefficient is not constant, but depends on the local Reynolds number, and the resolution of the turbulent integral-length scales (Meyers \& Sagaut 2006; Voke 1996). Based on the dynamic procedure (Germano 1992), $C_{s}$ can be adapted in space and time during a simulation (Germano et al. 1991). However, a correct dependence on the Reynolds number and the resolution of the integral-length scale is not guaranteed (Meneveau \& Lund 1997; Porté-Agel, Meneveau \& Parlange 2000).

Discretization of the LES equations further complicates the matter. It has been demonstrated that modelling and discretization errors strongly interact, and are difficult to separate (Ghosal 1996; Vreman, Geurts \& Kuerten 1996; Geurts \& Fröhlich 2002; Meyers, Geurts \& Baelmans 2003). In a pragmatic approach to this problem, it was shown that optimal settings (leading to minimal simulation errors) of the Smagorinsky coefficient $C_{s}$ can be determined (Meyers et al. 2003). This approach has been refined by evaluating a range of simulation errors simultaneously, leading to the identification of multi-objective parameter regions (Meyers, Sagaut \& Geurts 2006). Moreover, simulation errors increase rapidly when model coefficients are selected outside the multi-objective optimal regions. Hence, a good selection of the Smagorinsky coefficient is important, but difficult in practice. This is further substantiated by the fact that the dynamic procedure does not guarantee an optimal setting of the Smagorinsky model (Meyers et al. 2005, 2006), with dynamic errors which can be a posteriori twice as large as the minimal error levels. 
Reliability of LES results is usually evaluated through an extensive set of validation cases. However, in the absence of an experimental or direct numerical simulation (DNS) reference solution, validation is not always an option. Moreover, in the absence of the exhaustive LES database, such as employed by Meyers et al. (2006), the selection of the model coefficient $C_{s}$ and the corresponding simulation quality are uncertain. In these cases, confidence in simulation results is typically founded on a mix of common sense and an extensive sensitivity analysis of parameters which might affect simulation results. The source of variable parameters that can be thought of as uncertain, and which require attention, can be initial or boundary conditions (wall roughness, turbulent inflow parameters, etc.), material properties, source and interaction terms, geometry, etc. Such types of uncertainty are ubiquitous in engineering applications, and are often modelled as random processes. Less typical is the study of the effect of the model coefficients which are not always well-known from a theoretical point of view. In the present study, we address the sensitivity of LES to parametric uncertainties in the Smagorinsky (1963) model, more specifically the sensitivity to the coefficient $C_{s}$ that controls this model. To this end, we introduce techniques used in nonlinear uncertainty analysis to support the evaluation of parameter sensitivity and uncertainty. It is important to note that the occurrence of uncertain parameters in a computational model yields a change in the definition of the problem: instead of looking for the unique solution of a single fully deterministic problem, one is now interested in parameterizing the space of all solutions spanned by the uncertain parameters. The sensitivity of the solution with respect to a parameter is therefore related to the local value of the Jacobian of the solution in this space.

Clearly, a sensitivity analysis may not be expected to yield a direct evaluation of the above-discussed error sources and the different effects which determine correct settings of $C_{s}$. But, it is the only approach which is feasible in many practical engineering applications. Therefore, an assessment of the strengths and limitations of such an approach for large-eddy simulation, where the effect of modelling and discretization errors are difficult to gauge, is very useful. Further, the evaluation of $C_{s}$ as a stochastic parameter may provide an estimation of the variation in LES predictions resulting from the uncertainty in the model coefficient. We will demonstrate that large differences exist in the obtained probability density functions (p.d.f.s) for the large and small scales. Moreover, in the present simulations, $C_{s}$-invariant scales are detected, which are seen to separate the large scales and the small scales in the solution. For large values of $C_{s}$ these $C_{s}$-invariant scales disappear.

Uncertainty quantification requires the propagation of uncertainty through a given model, and affects all stages of the numerical simulation. Non-statistical stochastic approaches are available to efficiently treat stochastic partial differential equations. Spanos \& Ghanem (1989) pioneered the computational use of the Polynomial Chaos (PC) expansion method, which is based on the 'homogeneous chaos' theory of Wiener (1938), and is well-suited to solve stochastic differential equations. Wiener was the first to define homogeneous chaos as the span of Hermite polynomial functionals of a Gaussian process. According to the Cameron-Martin theorem (Cameron \& Martin 1947): "any nonlinear functional of a brownian motion can be represented with mean-square convergence as a Wiener-hermite series". As pointed out by one of the reviewers, the importance of this theorem is that it extends obvious results from finite-dimensional approximations (where various polynomials are complete with respect to their respective measures), to an infinite-dimensional context (Brownian motion instead of a finite set of random variables). Except for the Poisson and binomial measure (Schoutens 1999), there is no parallel result for general measures. 
Spanos \& Ghanem (1989) have successfully applied PC to various problems subject to Gaussian uncertainties in solid mechanics (Ghanem \& Spanos 1991).

The PC representation is a spectral decomposition of a second-order random process in terms of orthogonal basis functions. The spatial and temporal evolutions of the basis coefficients provide quantitative estimates of the modelled random process solution. The efficiency of this approach depends crucially on the judicious choice of 'coordinates' in probability space. Fast convergence in PC expansions implies that the polynomial basis on which PC expansions of the random solution are sought should be optimized to the statistics of the input data and of the random solution. The dimensionality of the representation, i.e. the number of terms required in the truncated PC expansion, grows rapidly as function of the number of random sources and their nature, and the degree of nonlinearity of the problem. The representation of a very 'noisy' random process (e.g. white noise) will theoretically require an infinity of terms. In this study, we use the generalized Polynomial Chaos (gPC) method (Xiu \& Karniadakis 2002) which has the advantage that Gaussian and non-Gaussian random processes can be represented. We emphasize that even if the gPC method does not provide a rigorous probabilistic framework to extend Wiener's theory to general measures, it has been successfully used to solve stochastic differential equations relying on finite-dimensional approximations (Xiu \& Karniadakis 2002; Lucor \& Karniadakis 2004b; Wan \& Karniadakis 2006). Moreover, it often allows a highorder deterministic approximation of random fields and appears to exhibit spectral convergence in many cases.

PC-based methods have been applied to different flow problems, such as porous media flows (Ghanem \& Dham 1998; Zhang \& Lu 2004), thermal problems (Hien \& Kleiber 1997; Hien \& Kleiber 1998; Xiu \& Karniadakis 2003b), and combustion. However, fewer studies exist that deal with full stochastic incompressible Navier-Stokes equations, see the review in Knio \& Le Maître (2006). Le Maître et al. (2001, 2002) have derived and implemented a stochastic Navier-Stokes PC solver using finite differences to investigate laminar fluid flow and transport problems. Xiu \& Karniadakis (2002) have generalized the approach to other non-Gaussian types of randomness and polynomials, and have applied it to incompressible stochastic two-dimensional flows (Xiu \& Karniadakis 2003a). Lucor (2004) has used the approach for three-dimensional flows as well. All of these have used spectral/hp element methods to solve the large time- and spacedependent deterministic PDE system produced by the Galerkin projection of the gPC method. Asokan \& Zabaras have developed a two-dimensional stabilized finiteelement stochastic formulation by considering an extension of the deterministic variational multi-scale approach with algebraic subgrid-scale modelling for the stochastic advection and the incompressible stochastic Navier-Stokes equations (Asokan \& Zabaras 2005b), and applied it to natural convection problems (Asokan \& Zabaras 2005a). While most of the PC-based studies have dealt with stochastic input generated by a finite number of random variables, Hou et al. (2006) have considered stochastic Burgers and two-dimensional Navier-Stokes equations (in a stream function-vorticity formulation) driven by Gaussian Brownian motion. They have introduced a PC compression technique similar to the sparse tensor products approach developed by Schwab (Frauenfelder, Schwab \& Todor 2005) to handle the constant flux of new random variables due to the Brownian motion and they obtained satisfactory results for short-time integration.

Nevertheless, many challenges remain to be resolved for the $\mathrm{PC}$ and $\mathrm{gPC}$ representations of complex and highly nonlinear processes. This is particularly true 
for cases where the stochastic solution does not depend smoothly and continuously on the random inputs, or for long-time integration of stochastic systems characterized by a limit-cycle oscillation response. For these cases, it was noticed that a spectral decomposition of the solution in terms of a global basis exhibits severe limitations. Different solutions have been proposed to mitigate those effects, such as an adaptive truncation strategy (Li \& Ghanem 1998; Lucor \& Karniadakis 2004a; Hou et al. 2006), or an adaptive decomposition based on a local basis combined with local refinement techniques (Le Maître et al. 2004; Wan \& Karniadakis 2005). In Le Maître et al. (2004), a multi-wavelet-based decomposition (Wiener-Haar) is proposed while in Wan \& Karniadakis (2005) an adaptive multi-element gPC is formulated improving the effectiveness of the gPC representation, as exemplified for the Kraichnan-Orszag three-mode problem (Orszag \& Bissonnette 1967).

There have been several attempts to apply the PC approach to turbulence. This was suggested in the early work of Wiener in 1939, but the idea lay dormant for twenty years. During the 1960s, several proposals were made to develop a theory of turbulence involving a truncated Wiener-Hermite expansion of the velocity field (by, among others, Orszag \& Bissonnette 1967; Meecham \& Jeng 1968; Crow \& Canavan 1970; Canavan 1970; Chorin 1974). The Hermite polynomial basis was used thanks to a quasi-normal hypothesis. All these works failed in the sense that the truncated expansion yields non-physical kinetic energy spectra. The main reason is that, owing to the non-local and nonlinear character of turbulence, a truncated PC approach is not able to account for all couplings and to describe correctly the kinetic energy transfers among modes and their correlations. Owing to its chaotic nature, a very large number of degrees of freedom are excited by the turbulent dynamics and the $\mathrm{PC}$ expansion is observed to converge very slowly. A consequence is that a direct numerical simulation of developed turbulence on the PC basis would necessitate the use of a unreasonably high-order polynomial basis, resulting in a untractable computational model. Some surrogate models have been developed to account for the effects of unresolved modes, but none of them yield fully satisfactory results. Therefore, the direct decomposition of the instantaneous turbulent field into a PC approach cannot at present be considered as an efficient way to address the issue of the sensitivity of a simulated turbulent flow. In the present study, we examine the use of polynomial chaos for the study of parametric uncertainty in large-eddy simulations. Though LES solutions do not formally correspond to Navier-Stokes turbulence, the governing equations are akin, such that the same convergence problems associated with the decomposition of the instantaneous LES field into a PC approach may be expected. What is proposed here is to preclude the problem mentioned above by considering the statistical moments of the simulated LES field (or related quantities such as the kinetic energy spectrum) as functions of the uncertain parameters, instead of decomposing the resolved turbulent velocity field itself. It will be shown that this approach makes it possible to obtain a fast-converging PC expansion of all statistical moments that are usually of interest, leading to a statistical parameterization of the space of the LES solutions spanned by the uncertain parameters under consideration.

We will address the sensitivity of LES to parametric variations in the subgrid-scale model. We choose the extensively documented Smagorinsky (1963) model, and we consider decaying homogeneous isotropic turbulence to illustrate the main principles. Although the Smagorinsky model is far from being the most advanced subgridscale model for LES, it has been studied extensively, which makes it a particularly interesting model to demonstrate our uncertainty approach. As well as the sensitivity analysis discussed above, we will also include, as an independent point of reference, 
comparisons with a direct numerical simulation. This will allow us to assess the value and limitations of conclusions from the sensitivity analysis. However, in view of the above discussion on the different error sources and contributions to model uncertainty, it is not the aim of the present study to provide a full DNS-based decomposition of these different contributions.

The paper is organized as follows. First, we present in $\S 2$ an overview of the sensitivity methodology that will guide us in our study. Next, in $\S 3$, we present the results of this methodology when applied to the analysis of the Smagorinsky model. Finally, our conclusions are summarized in $\S 4$.

\section{An overview of the sensitivity methodology}

First, in § 2.1, some LES fundamentals are briefly introduced, and the computational set-up is discussed. Next, in $\S 2.2$, we will detail the methodology which will be employed for the advanced sensitivity analysis.

\subsection{LES fundamentals and simulation set-up}

The dimensionless filtered Navier-Stokes equations for incompressible flows are given by

$$
\frac{\partial \bar{u}_{i}}{\partial t}+\frac{\partial \bar{u}_{i} \bar{u}_{j}}{\partial x_{j}}+\frac{\partial \bar{p}}{\partial x_{i}}-\frac{2}{R e} \frac{\partial \bar{S}_{i j}}{\partial x_{j}}-\frac{\partial \tau_{i j}}{\partial x_{j}}=0 \quad \text { with } \quad i=1,2,3
$$

where $\bar{u}_{i}$ is the filtered velocity component in the $x_{i}$-direction, $\bar{p}$ the filtered pressure and $R e$ the computational Reynolds number. The LES filter is denoted by $\overline{(\cdot)}$, and $\bar{S}_{i j}=\left[\partial \bar{u}_{i} / \partial x_{j}+\partial \bar{u}_{j} / \partial x_{i}\right] / 2$ corresponds to the filtered strain tensor. The filtering of the convective terms in the Navier-Stokes equations gives rise to the subgrid-scale stress tensor $\tau_{i j}$ given by

$$
\tau_{i j}=\overline{u_{i} u_{j}}-\bar{u}_{i} \bar{u}_{j}
$$

In LES, these subgrid-scale stresses are replaced by a model $m_{i j}$, which approximates their dynamic effect and is based on the resolved velocity field $\bar{u}_{i}$ only.

One of the most often employed formulations for $m_{i j}$ is the Smagorinsky (1963) model, which approximates the deviatoric part of $\tau_{i j}$ as

$$
m_{i j}=-2\left(C_{s} \Delta\right)^{2}|\overline{\boldsymbol{S}}| \bar{S}_{i j},
$$

with $C_{s}$ the Smagorinsky coefficient, $\Delta$ the LES filter width, and $|\overline{\mathbf{S}}|=\left(2 \bar{S}_{i j} \bar{S}_{i j}\right)^{1 / 2}$ the magnitude of the filtered strain-rate tensor.

In all simulations a second-order cell-centred finite-volume method is employed to discretize the closed LES equations This is combined with a four-stage second-orderaccurate Runge-Kutta time integration. LES of decaying homogeneous isotropic turbulence is carried out at a number of resolutions and different values for the model parameter $C_{s}$, and a Taylor-scale-based Reynolds number $R e_{\lambda}=100$. The initial fields for the LES are generated by filtering initial DNS fields taken from Meyers et al. (2003) with a sharp cutoff filter, with cutoff related to the grid cutoff wavenumber. During the simulations, no additional explicit filtering is performed and for the implementation of the Smagorinsky model, we further take $\Delta=h$.

\subsection{Space of possible solutions, sensitivity analysis and Polynomial Chaos}

We address the sensitivity of LES to parametric uncertainties in the subgrid-scale model. More specifically, we focus on the Smagorinsky parameter $C_{s}$ that controls the Smagorinsky model. Various $C_{s}$ distribution types and supports are considered. 
We use the generalized Polynomial Chaos method as a numerical tool to quantify the effect of the $C_{s}$ uncertainty on the LES solution. We first briefly recall the different stochastic approaches that are available before describing generalized Polynomial Chaos and its advantages.

\subsubsection{Statistical versus non-statistical stochastic approaches}

For the numerical solution of stochastic differential equations, we broadly distinguish statistical and deterministic methods.

The statistical approach includes among others Monte Carlo simulation, stratified sampling, and Latin hypercube sampling (Fishman 1996). These methods are straightforward to implement but the simulations can become prohibitively expensive, especially for systems that are already computationally complex even in their deterministic version.

Alternatively, one can develop deterministic methods for stochastic PDEs. The most widely used is the perturbation method, where input random fields are represented as infinite perturbation expansions of fluctuations around their mean fields. In practical applications, at most second-order expansions are employed (Kleiber \& Hien 1992; Liu \& Der Kiureghian 1991; Vanmarcke \& Grigoriu 1983; Zhu \& Wu 1991). A related approach is based on manipulation of the stochastic operators. Such methods include the Neumann expansion, which is based on expanding the inverse of the stochastic operator in a Neumann series (Shinozuka \& Deodatis 1988; Zhu, Ren \& Wu 1992), and the weighted integral method (Deodatis 1991; Deodatis \& Shinozuka 1991). But these methods have limitations and are restricted to small uncertainties.

Another approach to deterministic numerical solution of stochastic PDEs is based on introducing geometry and coordinates in the probability space on which input and solution uncertainty are modelled. The efficiency of this approach depends crucially on the judicious choice of 'coordinates' in probability space. Spanos \& Ghanem (1989) pioneered the computational use of the Polynomial Chaos (PC) expansion method, and have successfully applied it to various problems in solid mechanics (Ghanem \& Spanos 1991). PC expansions are based on the homogeneous chaos theory of Wiener (1938) and they allow a high-order deterministic approximation of random fields and appear to exhibit spectral convergence in many cases.

Thus, generalized PC expansions, also including the so-called Wiener-Askey chaos expansions, that are orthogonal with respect to non-Gaussian probability measures were first employed in computational algorithms by Xiu \& Karniadakis (2002), following developments in probability by Ogura (1972) and Schoutens (1999), and in orthogonal polynomials by Askey \& Wilson (1985) and Koekoek \& Swarttouw (1998). In gPC, the polynomials are chosen from the hypergeometric polynomials of the Askey family where the underlying random variables are not restricted to Gaussian random variables.

\subsubsection{Generalized Polynomial Chaos}

The generalized Polynomial Chaos (gPC) method is a non-statistical method used to solve stochastic differential (SDE) and stochastic partial differential equations (SPDE) (Spanos \& Ghanem 1989). It has recently emerged as a reliable and efficient numerical tool for numerous applications (Xiu \& Karniadakis 2003b; Ghanem \& Hayek 2002; Ghanem 1999; Ghanem \& Brzakala 1996; Ghanem \& Red-Horse 1999; Lucor \& Karniadakis 2004b). In the following, the gPC framework will be briefly described and Ghanem \& Spanos (1991) contains more details on the subject. 
Stochastic mathematical models are based on a probability space $(\Omega, \mathscr{A}, \mathscr{P})$ where $\Omega$ is the event space, $\mathscr{A} \subset 2^{\Omega}$ its $\sigma$-algebra, and $\mathscr{P}$ its probability measure.

Consider a random field $X(\omega)$, i.e. mappings $X: \Omega \rightarrow V$ from the probability space into a function space $V$. If $V=\mathbb{R}, X(\omega)$ are random variables, and if $V$ is a function space over a time and/or space interval, random fields are stochastic processes. $V$ is a Hilbert space with dual $V^{\prime}$, norm $\|\cdot\|$ and inner product $(\cdot, \cdot): V \times V \rightarrow \mathbb{R}$. As $V$ is densely embedded in $V^{\prime}$, we abuse notation and denote by $(\cdot, \cdot)$ the $V \times V^{\prime}$ duality pairing also.

We will consider second-order random fields, i.e. $X: \Omega \rightarrow V$ is a second-order random field over $V$, if

$$
\mathbb{E}\|X\|^{2}=\mathbb{E}(X, X)<\infty,
$$

where $\mathbb{E}$ denotes the expectation of a random variable $Y \in L^{1}(\Omega, \mathbb{R})$, and is defined by

$$
\mathbb{E} Y=\int_{\omega \in \Omega} Y(\omega) \mathrm{d} P(\omega) .
$$

Generalized polynomial chaos is a means of representing second-order random fields $X(\omega)$ parametrically through a set of random variables $\left\{\xi_{j}(\omega)\right\}_{j=1}^{N}, N \in \mathbb{N}$, through the events $\omega \in \Omega$ :

$$
X(\omega)=\sum_{k=0}^{\infty} a_{k} \Phi_{k}(\xi(\omega)) .
$$

Here $\left\{\Phi_{j}(\xi(\omega))\right\}$ are orthogonal polynomials in terms of a zero-mean random vector $\xi:=\left\{\xi_{j}(\omega)\right\}_{j=1}^{N}$, satisfying the orthogonality relation

$$
\left\langle\Phi_{i} \Phi_{j}\right\rangle=\left\langle\Phi_{i}^{2}\right\rangle \delta_{i j}
$$

where $\langle\cdot, \cdot\rangle$ denotes the ensemble average. We note that the modal coefficients $a_{k}$ are purely deterministic quantities. The number of random variables $N \in \mathbb{N}$ is in general infinite, so is the index in (2.4). In practice, however, we need to retain a finite set of random variables, i.e. to $\left\{\xi_{j}\right\}_{j=1}^{N}$ with $N<\infty$, and a finite-term truncation of (2.4).

The inner product in (2.5) is based on the measure $W(\xi)$ of the random variables:

$$
\langle f(\xi) g(\xi)\rangle=\int_{\omega \in \Omega} f(\xi) g(\xi) \mathrm{d} P(\omega)=\int f(\xi) g(\xi) W(\xi) \mathrm{d} \xi,
$$

with $W(\xi)$ denoting the density of the law $\mathrm{d} P(\omega)$ with respect to the Lebesgue measure $\mathrm{d} \xi$ and with integration taken over a suitable domain, determined by the range of $\boldsymbol{\xi}$.

In equation (2.4), there is a one-to-one correspondence between the type of the polynomial $\{\Phi\}$ and $W(\xi)$. Indeed, the weighting function of $\{\Phi\}$ has a similar form to $W(\xi)$. For instance, the weighting function of Hermite orthogonal polynomials corresponds to $\exp \left(-\frac{1}{2} \xi^{T} \xi\right) /(2 \pi)^{n / 2}$, and is the same as the probability density function (p.d.f.) of the $N$-dimensional Gaussian random variables $\boldsymbol{\xi}$. Hence, the classical Wiener polynomial chaos is an expansion of Hermite polynomials in terms of Gaussian random variables. A correspondence between orthogonal polynomials and random variables was first established by Ogura (1972) and Schoutens (1999).

The expansion in equation (2.4) is truncated to a finite-dimensional space based on a 'finite-dimensional noise assumption' that is accomplished by characterizing the probability space by a finite number $N$ of random variables. Further, the highest order $P$ of the polynomials $\{\Phi\}$ is selected based on accuracy requirements. Consequently, 
the finite-term expansion takes the form

$$
X(\omega)=\sum_{j=0}^{M-1} a_{j} \Phi_{j}(\xi(\omega)),
$$

where $\xi=\left(\xi_{1}, \ldots, \xi_{N}\right)^{T}$ is an $N$-dimensional random vector with $\xi_{i}$ independent of $\xi_{j}$ for all $1 \leqslant i \neq j \leqslant N$. If we denote the highest order of polynomial $\{\Phi\}$ as $P$, then the total number of expansion terms $M$ is (Ghanem \& Spanos 1991),

$$
M=(N+P) ! /(N ! P !) .
$$

The multi-dimensional gPC expansion is constructed as the tensor product of the corresponding one-dimensional expansion. Note that in one-dimensional expansions $(N=1)$, we have $M=P+1$.

\subsubsection{Non-intrusive formulation of the problem}

For our application, the Smagorinsky constant $C_{s}$ takes the role of the uncertain parametric quantity. We treat $C_{s}$ as an uncertain input to the stochastic problem, and its probability distribution is assumed. In order to simplify the system, we make the additional assumption than $C_{s}$ is a random variable, which means that its statistical properties do not depend on spatial or temporal dimensions. Therefore, $N=1$ in (2.8) and only two terms in $\xi(\omega)$ (zero- and first-order term) are needed to represent $C_{s}$ exactly. Because of the dependence of the LES solution on the uncertain parameter $C_{s}$, any physical quantity part of (or derived from) the LES solution becomes a random process itself. This applies for instance, to the turbulent kinetic energy spectrum, $E(k, t)$, and it can then be expressed as

$$
E(k, t, \omega)=\sum_{j=0}^{M-1} e_{j}(k, t) \Phi_{j}\left(C_{s}(\omega)\right) .
$$

We emphasize that this explicit representation of the solution (or derived quantity) as a function of $C_{s}$ holds if the distribution of $C_{s}$ is known and if the $M$ coefficients $e_{j}(k, t)$ have been computed.

In order to compute those coefficients, there exists a so-called 'intrusive' approach of the gPC application (Le Maître et al. 2001; Xiu \& Karniadakis 2003a). It is based on the substitution of the gPC expansion (2.4) into the SDE in order to represent both the solution and the random inputs. The new system is then projected (with a Galerkin-type projection) onto the orthogonal polynomial basis. This leads to a deterministic coupled system of $M$ differential equations, the coefficients $e_{j}$ being solutions of that system. This approach will not be discussed further in this study, and we should point out that it requires substantial modifications of the existing deterministic solver.

Another so-called 'non-intrusive' approach of the gPC application consists of projecting the stochastic solution directly onto each member of the orthogonal basis chosen to span the random space (Webster, Tatang \& McRae 1996; Tatang et al. 1997). In order to do so, a set of deterministic solutions is first generated. The projection is analogous to a collocation method, as opposed to the Galerkin method, in the sense that it requires knowledge of the solutions at some discrete quadrature points. This approach is generally much more efficient than brute-force sampling methods such as Monte-Carlo-based methods. Moreover, the non-intrusive approach does not require any substantial modifications to the existing deterministic solver. 
This approach will be used in this study. The $e_{j}$ random coefficients can be directly computed as they take the following form:

$$
(\forall j \in\{0, \ldots, M-1\}) \quad e_{j}(k, t)=\frac{\left\langle E(k, t, \omega) \Phi_{j}\left(C_{s}(\omega)\right)\right\rangle}{\left\langle\Phi_{j}^{2}\left(C_{s}(\omega)\right)\right\rangle} .
$$

We recall that $\left\langle\Phi_{j}\right\rangle=0$ for $j>0$, and the denominator $\left\langle\Phi_{j}^{2}\right\rangle$ can be tabulated prior to the projection. The evaluation of (2.10) is equivalent to computing multidimensional integrals over the domain $\Omega$ (2.6). Different ways of dealing with highdimensional integrations can be considered depending on the relative prevalence of accuracy and efficiency (Keese 2005). Here, we use a numerical quadrature of Gauss or Gauss-Lobatto type by full tensor products. This approach remains computationally efficient for moderate $N$. The number of quadrature points $N_{q}$ to use depends on the regularity of the function to integrate. We emphasize that the deterministic solver will compute/provide the value of $E(k, t)$ at those known quadrature points and not at randomly selected locations.

After solving for the deterministic coefficients $e_{j}$, we have an explicit functional representation (in random space) of the solution process. It is then possible to explicitly reconstruct the response surface of the random process $E(k, t)$ by drawing a population of samples $C_{s}$ according to their probability distribution (2.4). This reconstruction is trivial and computationally fast.

Moreover, it is also possible to perform a number of analytical operations on the stochastic solution. Moments, sensitivity analysis, confidence intervals and the p.d.f. of the solution can be evaluated. Owing to the orthogonality of the modes, the moments can be easily computed. The mean solution is contained in the expansion term with zero-index. The second moment, i.e. the covariance function is given by a linear combination of the modal fluctuations. We have

$$
\left.\begin{array}{l}
\mu_{E(k, t)}=\langle E(k, t, \omega)\rangle=e_{0}(k, t) \\
\sigma_{E(k, t)}^{2}=\left\langle\left(E(k, t, \omega)-e_{0}(k, t)\right)^{2}\right\rangle=\sum_{j=1}^{M-1}\left[e_{j}^{2}(k, t)\left\langle\Phi_{j}^{2}\right\rangle\right] .
\end{array}\right\}
$$

Note that, once the response surface for given random input data is determined, we can determine the statistics of the solution for any random input with different statistics (Knio \& Le Maître 2006). This approach performs well as long as the initial response surface is sufficiently accurate, and provided the new data event space $\Omega$ is the same as the previous one. In this case, this leads to significant computational savings since the associated sampling costs are substantially smaller than those required to determine the original stochastic solution.

\section{The sensitivity methodology applied to the Smagorinsky model}

First, in $\S 3.1$, a detailed overview of the LES results and error behaviour is presented, using DNS results as a reference. These results illustrate the typical variations in LES results associated with changes in the Smagorinsky coefficient or the resolution. They provide a useful reference framework, which will be used to better understand and interpret the trends which are observed in the effective sensitivity analysis in $\S 3.2$ and §3.3. In §3.2, no a priori knowledge is assumed on the relevant range of the coefficient, but rather we will investigate whether and how sensitivity analysis can help to select a $C_{s}$ range. Finally, in $\S 3.3$ the effect of the $C_{s}$-distribution on the LES probability density functions is considered. 


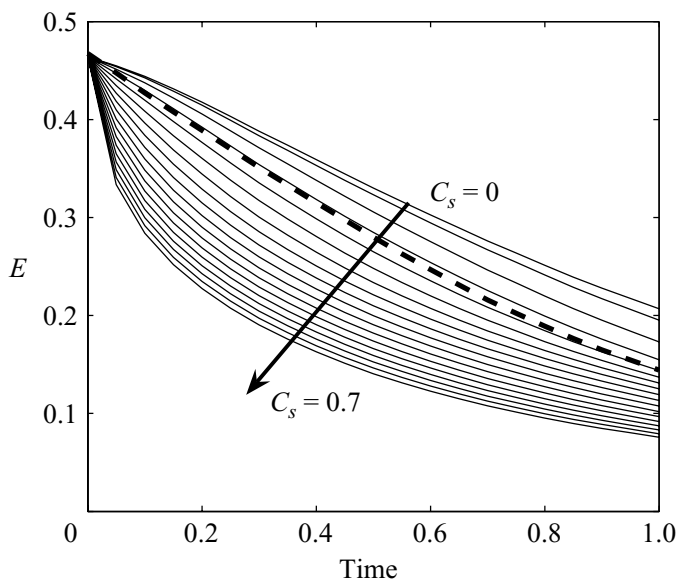

FIGURE 1. Energy decay of $32^{3}$ LES with Smagorinsky constants ranging between 0 and 0.7 . - , LES results; --, filtered DNS results.

\subsection{LES sensitivity for a wide range of $C_{s}$ values}

A good selection of $C_{s}$ values depends on several factors such as the simulation Reynolds number, the resolution, and the numerical implementation. Experience dictates that a sensible selection for $C_{s}$ ranges from 0 to 0.2. Though the Smagorinsky model and its coefficient $C_{s}$ are subjects of a large number of studies in the literature, this is not necessarily the case for other models. Therefore, in the present sensitivity study, we will try to avoid the use of a priori knowledge for the selection of a parametric range.

LES results are first evaluated for a wide range of $C_{s}$ values, and we select somewhat arbitrarily $0 \leqslant C_{s} \leqslant 0.7$, i.e. an interval which is considerably larger than commonly accepted. Based on the analysis of these results, and without the use of any external information (such as e.g. DNS data), a refinement of the $C_{s}$ interval is made. In the next sections, this smaller $C_{s}$ range will be used as our study interval for more detailed sensitivity analysis.

In figure 1, the decay of kinetic energy as function of time is presented for $0 \leqslant C_{s} \leqslant 0.7$ and a $32^{3}$ simulation. The filtered DNS results are also shown as a reference. Results demonstrate that the LES solution changes strongly with $C_{s}$. Clearly, if the DNS data are not taken into consideration, no obvious preference can be given to any of these solutions, and this illustrates a typical limitation which is often encountered in sensitivity analysis.

In order to further establish the dependence of the LES solution on $C_{s}$, the evolution of energy spectra as function of $C_{s}$ is presented in figure 2. To this end, spectra for $0 \leqslant C_{s} \leqslant 0.7$ are presented at $t=0.8$, i.e. near the end of the decay period considered in the present simulations. Results are shown for the $32^{3}, 48^{3}$, and $64^{3}$ resolutions, and several interesting features appear. First, for the small resolved modes in the solution $k \gg 1$, one can observe that the energy $E(k)$ in those modes decreases monotonically with $C_{s}$. In contrast, for the large scales $k \approx 1, E(k)$ typically first increases, and then decreases when $C_{s}$ increases from 0 to 0.7 .

For low to moderate values of $C_{s}\left(0 \leqslant C_{s} \lesssim 0.2\right)$, one can further observe in figure 2 that all spectra share a common point $\left(k \approx 5, k \approx 6\right.$, and $k \approx 9$ for $32^{3}, 48^{3}$ and $64^{3}$ respectively), at which the energy is independent of $C_{s}$. To the right of this point, we observe the above-discussed monotonic decrease of $E(k)$. To the left, $E(k)$ does not 

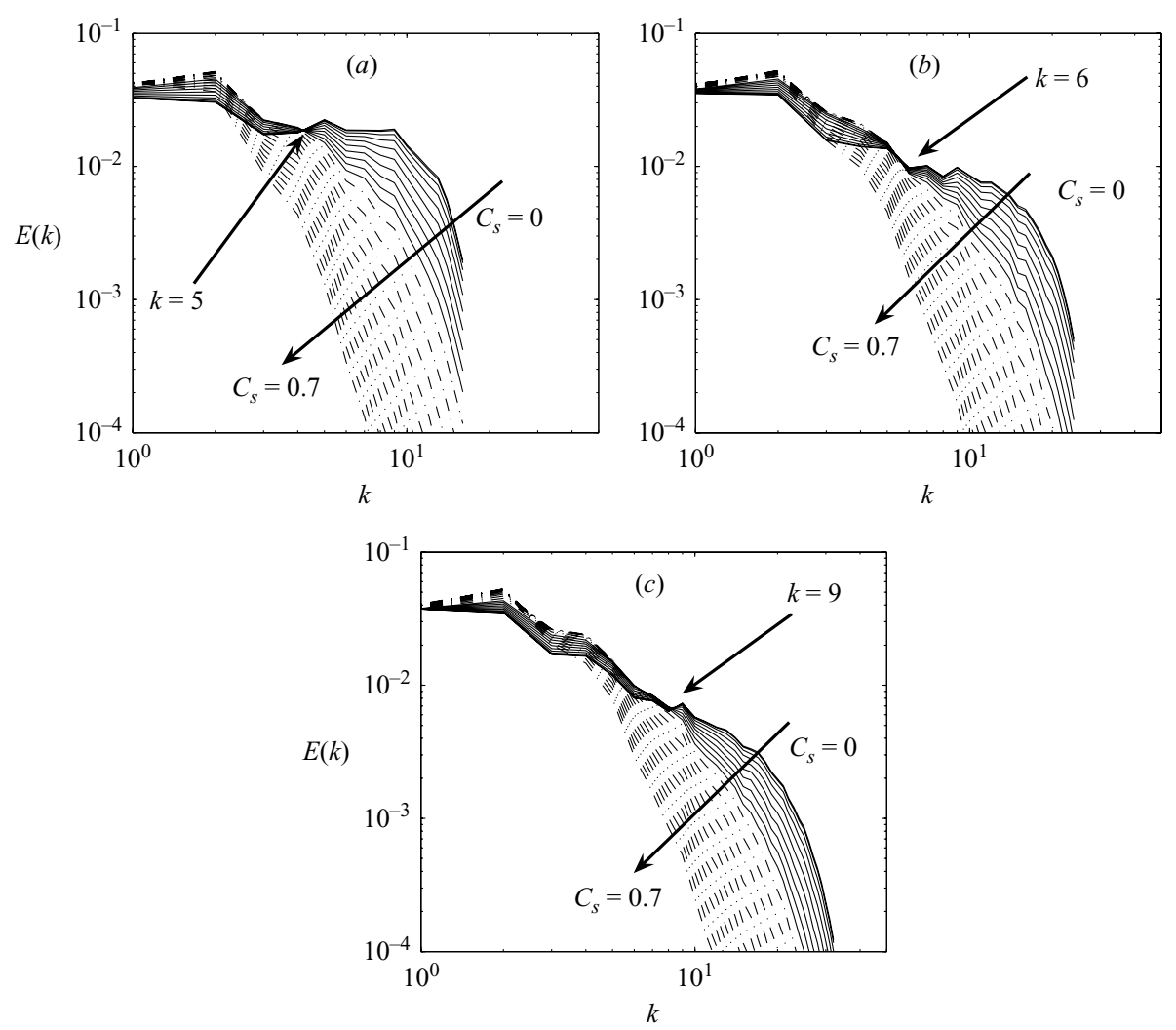

FIGURE 2. Energy spectra at $t=0.8$ of $(a) 32^{3}$, (b) $48^{3}$, and (c) $64^{3}$ LES with Smagorinsky constants ranging between 0 and 0.7 . - , low to medium $C_{s}$ range $\left(0 \leqslant C_{s} \lesssim 0.2\right) ;-\cdot-$, medium to high $C_{s}$ range $\left(0.2 \lesssim C_{s} \leqslant 0.7\right)$.

change monotonically with $C_{s}$. Obviously, for higher values of $C_{s}$, one can appreciate that the 'common point' disappears. These observations are intriguing and need some further discussion.

In high-Reynolds-number turbulence, it is well-known that the large-scale flow features are independent of the small-scale dissipation mechanism which converts the turbulent kinetic energy into heat (see e.g. Pope 2000; Frisch 1995). Hence, a change in the dissipation mechanism (e.g. small variations in the viscosity) is not reflected in the large scales but, rather, the small-scale energy distribution automatically adjusts, such that the overall turbulent kinetic energy balance is maintained. In LES, these properties allow, from a conceptual point of view, the classical NavierStokes dissipation mechanism to be replaced with a subgrid-scale closure. The LES aim is to reproduce the effect of the smallest turbulent scales in a computational more affordable way. Even though at marginal resolutions, and for 'imperfect' subgrid-scale models, this ideal separation between the dissipation mechanism and the large scales is not present, some of its features appear to remain. One of the few studies in this context, Jiménez (1999), showed that for Smagorinsky LES, slightly incorrect settings of $C_{s}$ will be counterbalanced by a change in the tail of the spectrum. Too low settings of $C_{s}$ result in an energy pile up at the tail of the spectrum, while too high values result in a tail which falls of too steeply. 
The observations in figure 2 can be seen in this context. For a reasonable range of $C_{s}$ settings $\left(0 \leqslant C_{s} \lesssim 0.2\right)$ the tail of the spectrum is adapted drastically. Because of the low simulation resolution, the variation of $C_{s}$ also is reflected on the large scales. However, their dependence on $C_{s}$ is weaker, and the transition to small-scale behaviour is sharply marked by the common point discussed above.

If results for $C_{s} \gtrsim 0.2$ in figure 2 are considered, one observes that spectra no longer include this common point. Hence, the clear demarcation between large and small scales disappears for higher $C_{s}$ values, and the full spectrum shows the properties of a dissipation range. We propose to use the value $C_{s}^{+}$, which separates the two regimes observed, i.e. LES spectra having a common point (low $C_{s}$ ) and not having such a point (high $C_{s}$ ), as an upper limit for a sound $C_{s}$ range.

\subsection{Sensitivity analysis: determination of an appropriate parametric range}

We now turn to an extensive sensitivity analysis of the current LES case to the parameter $C_{s}$ by means of generalized polynomial chaos. This provides an elegant and efficient way to reconstruct the $C_{s}$ dependence of the LES solution, and also allows quantification of how uncertainty in $C_{s}$ affects the LES results.

In the current section, $C_{s}$ is considered to follow a uniform distribution $U$, which is a particular case of a beta distribution, and $0 \leqslant C_{s} \leqslant 0.7$. We designate the distribution by $U(\alpha, \beta)$, where $\alpha$ is the mean value of the distribution and $\beta$ is half the width of the support. This distribution has a finite support and does not 'favour' any $C_{s}$ choice. We use the gPC method with an orthogonal basis of Legendre polynomials that are the most appropriate for this type of distribution (Xiu \& Karniadakis 2002). We emphasize that this choice is not indisputable. The optimality is only guaranteed for the stochastic inputs. For nonlinear problems, it is not clear that an optimal representation of the inputs is necessarily optimal for the entire problem.

Therefore, before turning to the effective presentation of sensitivity results, we will describe a brief convergence analysis of the gPC results. Two types of errors can affect the accuracy of the non-intrusive gPC representation. The first is the quadrature error that enters the computation of each random mode (2.10). The number of quadrature points required to reach a prescribed accuracy depends on the regularity of the product between the polynomial basis and the random process to be represented (which is not known a priori). The second error is the approximation error due to the truncation of the gPC expansion (2.9).

We briefly study the behaviour of each of these errors for our specific problem. We first look at the quadrature error for the computation of the mean solution. Subsequently, we investigate the approximation error in the reconstructed gPC solution. The convergence study is carried out for the $64^{3}$ case, taking a uniform distribution for $C_{s}$.

In figure 3(a), we present the convergence rate of the first gPC mode (or mean solution (2.11)) versus the number of Gauss-Lobatto quadrature points $N_{q}$. To this end, we evaluated the convergence of $L_{2}$ norms based on the energy spectra $E(k, t)$, representative of the convergence of large-scale flow features, and based on the enstrophy spectra $k^{2} E(k, t)$, which characterize the resolved small-scale part of the solution. The error (in percent) is relative to a very fine quadrature employing $N_{q}=70$ points. The curves decay exponentially fast and only few realizations are needed to get accurate results. For instance, only seven quadrature points are required to get both mean physical quantities within $0.1 \%$.

In figure $3(b)$, we present the convergence rate of the error of the gPC reconstructed solutions versus the order $P$ of the polynomial basis. Here, $N_{q}=16$ quadrature points 

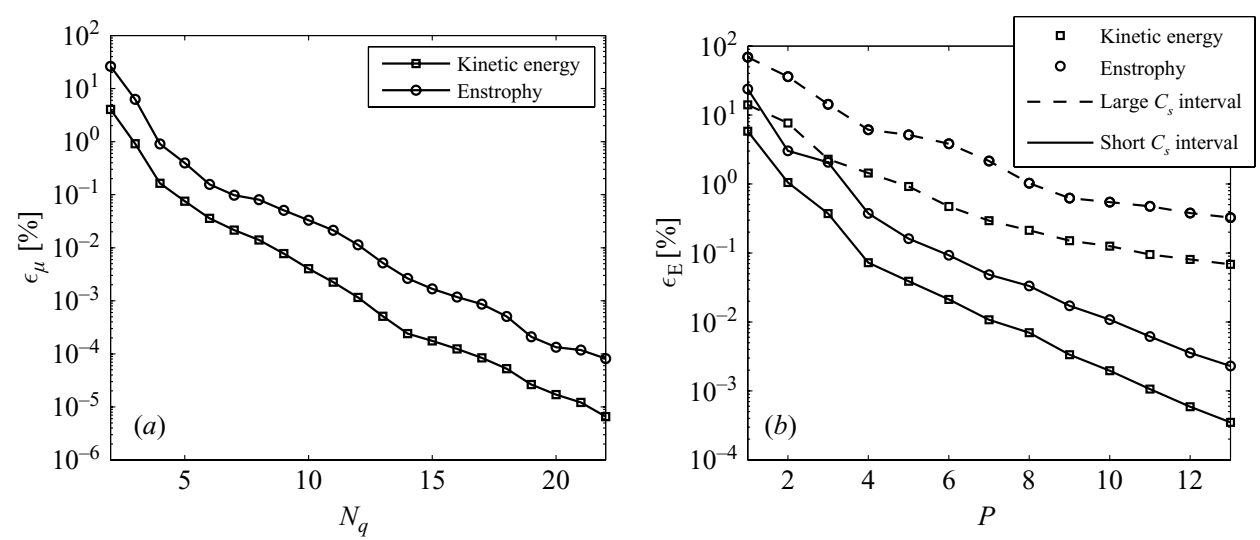

FIgURE 3. Convergence rates of the gPC representation for the $64^{3}$ case. (a) $L_{2}$ norms of the error in the mean kinetic energy and enstrophy spectra versus number of quadrature points; $C_{s}$ follows $U(0.35,0.35)$. (b) $L_{2}$ norms of the error in kinetic energy and enstrophy spectra versus polynomial order $P$. Two different Smagorinsky constant ranges are presented: large range: $C_{s}$ follows $U(0.35,0.35)$ and small range: $C_{s}$ follows $U(0.0702,0.0702)$.

have been used to produce the results. The error (in percent) is computed relative to the deterministic LES solution and depends on the resolution $N_{r}$ as

$$
\epsilon_{E}\left(N_{r}\right)=\left[\frac{\int_{0}^{k_{c}} \int_{\Omega}\left\{E_{g P C}\left(k, t, C_{s}(\omega)\right)-E_{L E S}\left(k, t, C_{s}(\omega)\right)\right\}^{2} \mathrm{~d} \omega \mathrm{d} k}{\int_{0}^{k_{c}} \int_{\Omega} E_{L E S}^{2}\left(k, t, C_{S}(\omega)\right) \mathrm{d} \omega \mathrm{d} k}\right]^{1 / 2},
$$

for the energy spectra $E(k, t)$, and a similar definition is used for the enstrophy spectra $k^{2} E(k, t)$. The wavenumber $k_{c}=\pi \mathscr{L} / N_{r}$ corresponds to the grid cutoff, and $\mathscr{L}$ is the computational box size.

The integrals over $\Omega$ in (3.1) are numerically evaluated over a discrete set of points $N_{p}$ distinct from the quadrature set of points $N_{q}$, e.g. $N_{p}=70$ points are used for the large $C_{s}$ range. Two different $C_{s}$ ranges are investigated. Both cases exhibit spectral convergence. As expected, it is computationally more costly to reach a given accuracy with a gPC representation for the large $C_{s}$ interval than the short one.

In the remainder of this paper, we will use a maximum of $N_{q}=22$ deterministic realizations in the interval $0 \leqslant C_{s} \leqslant 0.7$ and a corresponding order $P=20$, such that errors related to the $\mathrm{gPC}$ reconstruction and the quadrature rule are small.

We now return to the sensitivity analysis, and first elaborate on the determination of $C_{s}^{+}$. In order to easily determine $C_{s}^{+}$, one can display the energy in the first modes of the spectrum as function of $C_{s}$. After solving for the deterministic gPC coefficients (2.10), we reconstruct the energy distribution by drawing a population of samples $C_{s}$ according to their probability distribution (2.9). The results are shown in figure 4 . Obviously the energy in these modes changes non-monotonically with $C_{s}$, and hence, for low values of $k$, maxima can be observed which differ from $C_{s}=0$. Apart from mode $k=1$, where the maximum is presumably located at $C_{s}>0.7$, these maxima are marked on figure 4 . One can further observe that the $C_{s}$ value at which the energy reaches its maximum roughly decreases for smaller turbulent scales. For higher modes, the maximum energy $E\left(k, C_{s}\right)$ occurs at $C_{s}=0$, and also, for $C_{s} \gg 0$, 

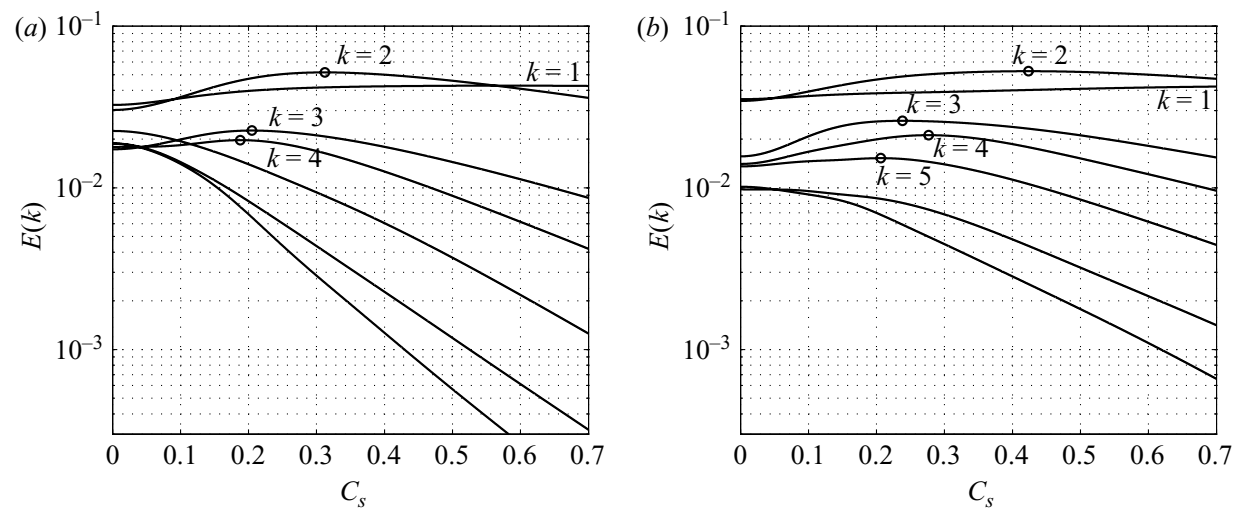

FiguRE 4. Turbulent kinetic energy distribution vs. $C_{s}$ with uniform distribution $U(0.35,0.35)$, for the largest turbulent scales. Circles show the maximum energy for each scale. (a) $32^{3}$ grid cells; (b) $48^{3}$ grid cells.

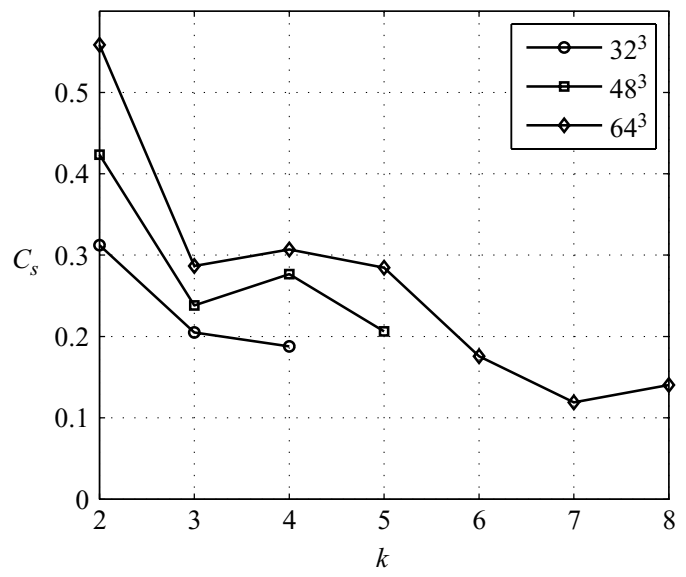

FIGURE 5. $C_{s}$ positions of maximum kinetic energy for the large turbulent scales and for three different grid sizes.

$E\left(k, C_{s}\right) \sim \exp \left(-C_{s}\right)$. Hence, these modes are in the small-scale part of the spectrum and change monotonically with $C_{s}$. A summary of these results is presented in figure 5, where the $\left(k, C_{s}\right)$ coordinates of these maxima are displayed for LES with $32^{3}, 48^{3}$ and $64^{3}$ resolutions. Hence, the last point of these curves (at highest $k$ ) provides an estimation for $C_{s}^{+}: C_{s}^{+}=0.1879$ for the $32^{3}$ resolution, $C_{s}^{+}=0.2064$ for the $48^{3}$ resolution and $C_{s}^{+}=0.1404$ for the $64^{3}$ resolution. Note that the dependence of $C_{s}^{+}$ on the resolution is not monotonic. However, in particular for marginal simulation resolutions, the dependence of LES results on the resolution can be quite irregular (see e.g. Meyers et al. 2006).

To further evaluate the relevance of $C_{s}^{+}$as an upper bound for a parametric uncertainty evaluation of $C_{s}$, the simulation error (using filtered DNS as reference data) is evaluated as function of $C_{s}$ in figure 6 . To this end, we define a simulation 


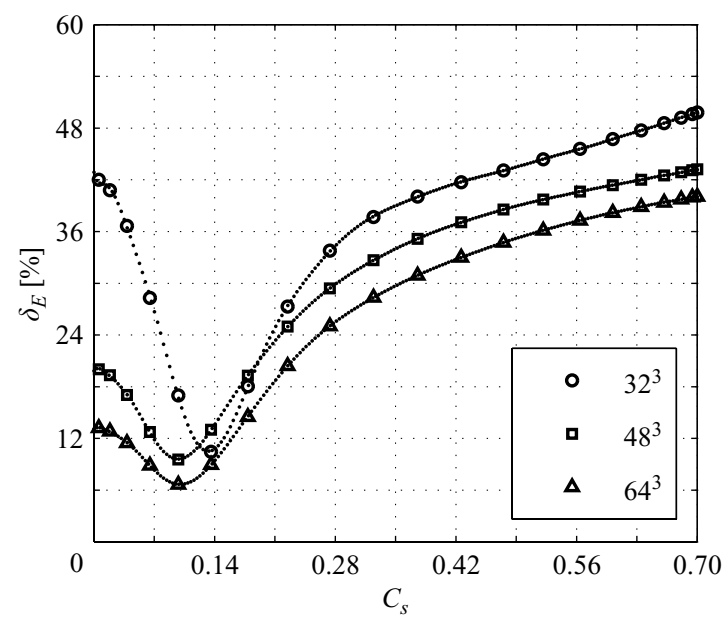

FIGURE 6. gPC reconstructed errors in resolved kinetic energy at $t=0.8$ of $32^{3}(\circ), 48^{3}(\square)$ and $64^{3}(\times)$ LES as a function of $C_{s}$.

error $\delta_{E}$ as a function of the resolution $N_{r}$ and the coefficient $C_{s}$ as

$$
\delta_{E}\left(N_{r}, C_{s}\right)=\left[\frac{\int_{0}^{k_{c}}\left\{E_{L E S}(k, t)-E_{D N S}(k, t)\right\}^{2} \mathrm{~d} k}{\int_{0}^{k_{c}} E_{D N S}^{2}(k, t) \mathrm{d} k}\right]^{1 / 2} .
$$

A more systematic approach to simulation errors in LES may be considered (see e.g. Meyers et al. 2006, and our discussion in $\S 1$ ), but does not shed further light on our present discussion. In figure 6 , we evaluated $\delta_{E}$ at $t=0.8$. The symbols represent the error between LES and DNS solutions at the different $C_{s}$ quadrature points; those points are chosen in accordance with a Gauss-type or Gauss-Lobatto-type quadrature rule (in which case they also include the boundary points: $C_{s}=0$ and $C_{s}=0.7$ ). The dotted lines (reconstructed gPC solution) represent the population of possible LES kinetic-energy-error events in the domain of uncertainty. Roughly speaking, it predicts how accurate the LES solution is compared to the DNS solution for a given $C_{s}$ value. One can see in the figure that the sharp gradients of the distributions around the optimal $C_{s}$ values are correctly captured by the method. Moreover, systematic Monte-Carlo samplings of the solution (not shown in the figure) at various $C_{s}$ values have been produced in order to check the accuracy of the gPC predictions. Very good agreement was obtained at those points between the gPC prediction and the deterministic computation.

It is now clear that $C_{s}^{+}\left(N_{r}\right)$ as obtained in figure 5 is indeed a good upper bound for all three resolutions considered (cf. figure 6). The existence of $C_{s}^{+}$, and its use as an upper bound for the model parameter, may be considered for other subgrid-scale closures as well, e.g. various model formulations have a parameter similar to the Smagorinsky coefficient (Meyers \& Sagaut 2006; Vreman 2004; Hughes, Mazzei \& Oberai 2001; Nicoud \& Ducros 1999). Further research is needed in order to establish whether this type of limit, and the related invariant point in the spectra, also appears for these subgrid-scale models.

We now show how the gPC approach can be used to visualize the space of LES solutions spanned by the uncertainty in the Smagorinsky constant $C_{s}$. Figure 7 shows 

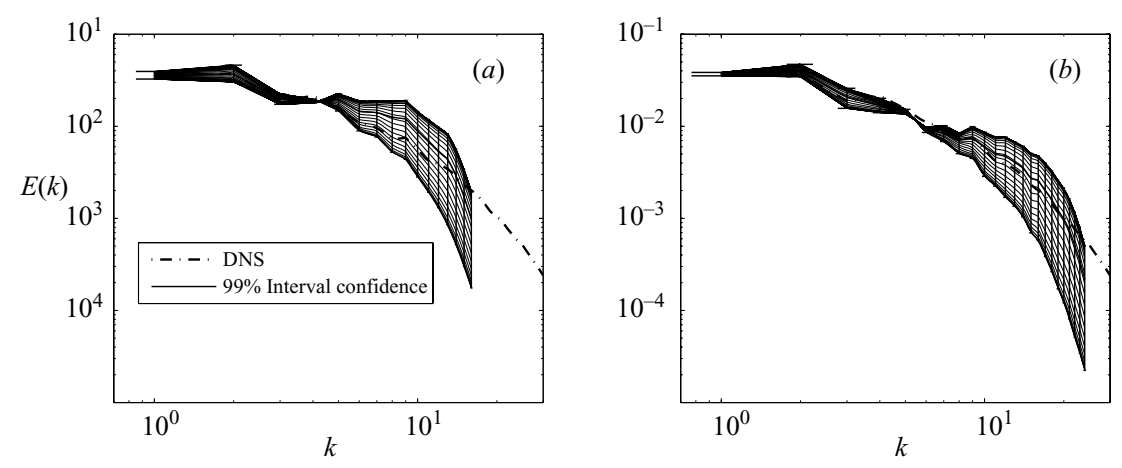

FiguRE 7. Turbulent kinetic energy spectrum with $99 \%$ confidence interval; $(a) C_{s}$ : uniform distribution $U(0.094,0.094)$ for the $32^{3}$ grid case; $(b) C_{s}$ : uniform distribution $U(0.1032,0.1032)$ for the $48^{3}$ grid case.

the turbulent kinetic energy spectra plotted with logarithmic axes and their associated $99 \%$ confidence intervals represented by error bars. The results are compared to the deterministic DNS reference data. The LES solution computed at the gPC quadrature points is shown as well in order to check that it lies within the envelop described by the confidence intervals. We recall that here the $C_{s}$ ranges have been reduced following previous discussions. For each turbulent scale, once the gPC coefficients of the energy have been computed, the stochastic response is then generated by drawing 500000 uniformly distributed samples of $C_{s}$. We verified that this sample size is sufficient for our application. The p.d.f. is then evaluated from the population ensemble. The p.d.f. results converge as we increase the level of representation of the kinetic energy through the polynomial order $P$ and the number of samples. Moreover, we note that convergence in the mean is fast but the convergence in the probability of the tails requires more effort.

\subsection{Sensitivity analysis: the effect of the distribution}

A more systematic study is pursued in order to investigate the effect of the type of probabilistic distribution of the parameter $C_{s}$ on the LES solution. After the uniform distribution, we naturally turn our attention to the beta distribution $B$ which has a finite support and that we designate by $B(\alpha, \beta)$ such that

$$
f(\xi)=\frac{\mathrm{I}_{[-1,1]}(\xi)}{2^{\alpha+\beta+1}} \times \operatorname{Beta}(\alpha+1, \beta+1) \times(\xi+1)^{\beta}(1-\xi)^{\alpha},
$$

where $\operatorname{Beta}(\cdot, \cdot)$ is the Beta function and the indicator function $\mathrm{I}_{[-1,1]}$ ensures that only values of $\xi$ in the range $[-1,1]$ have non-zero probability. Since the uncertain parameter $C_{s}$ is positive and taken within a certain range $[a, b]$, a change of variable is required and the probability distribution is scaled accordingly.

For this analysis, we keep the reduced intervals introduced in the previous section, i.e. $0 \leqslant C_{s} \leqslant C_{s}^{+}$. A natural choice is to pick the mean value of each distribution at the centre of the interval of variability. This choice is somewhat empirical but is the least biased when no additional information is available. We first consider symmetric distributions, which in this case imposes that $\alpha=\beta$, i.e. with zero skewness. Different distributions are considered, as represented in figure $8(a)$, with increasingly higher probability density around the mean value. The mean value $\mu_{B(\alpha, \beta)}=\frac{1}{2} C_{s} / C_{s}^{+}$is the same for each distribution but the variance decreases and the kurtosis increases as the $(\alpha, \beta)$ pair increases. The skewness of these distributions is zero. 

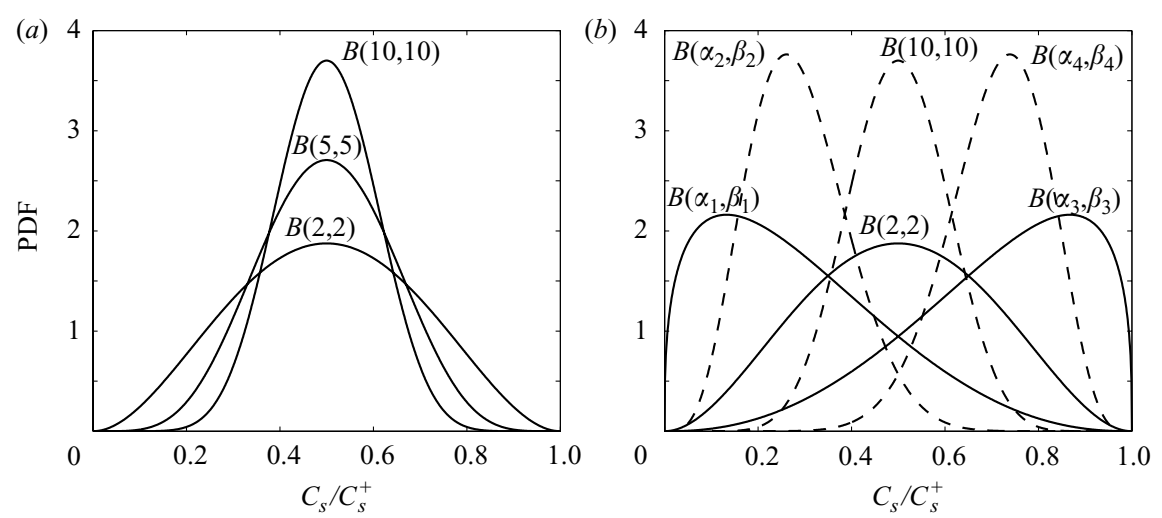

Figure 8. Beta distributions $B(\alpha, \beta)$ for the Smagorinsky constant $C_{s}$. The p.d.f.s have: (a) same mean $\mu_{B(\alpha, \beta)}=\frac{1}{2} C_{S} / C_{S}^{+}$, different variance $\sigma_{B(\alpha, \alpha)}^{2}$ and same skewness (zero value); (b) different mean, same variance $\sigma_{B(\alpha, \beta)}^{2}=\sigma_{B(2,2)}^{2}$ (solid lines) or $\sigma_{B(\alpha, \beta)}^{2}=\sigma_{B(10,10)}^{2}$ (dashed lines) and different skewness. Approximate values for the parameters are: $\alpha_{1}=\beta_{3}=0.36$, $\beta_{1}=\alpha_{3}=2.38, \alpha_{2}=\beta_{4}=4.14$ and $\beta_{2}=\alpha_{4}=11.72$.

A sensitivity analysis is performed by means of the gPC representation. We use an orthogonal basis of Jacobi polynomials that are most appropriate for beta distributions (Xiu \& Karniadakis 2002). The resulting probability density functions of the turbulent kinetic energy, obtained for each turbulent scale, due to the uncertainty in $C_{s}$ are shown in figure $9(a-c)$. This time a linear scale is used to represent the turbulent kinetic energy content. We notice that the turbulent kinetic energy response is finite for each scale. Moreover, the non-zero skewness of most of the distributions (as opposed to the input) emphasizes the nonlinearity of the phenomena. We can see that the energy distribution among the different scales follows a similar trend for the three cases.

In figure $10(a)$, we present the coefficient of variation $c_{v}$ of the turbulent kinetic energy in each scale. The coefficient of variation is a non-dimensional number and is a measure of dispersion of a probability distribution. It is defined, for each turbulent scale, as the ratio of the standard deviation and the mean. The coefficient of variation is relatively large for the large scales (except for the first one) with a sharp drop toward the scale that is insensitive to changes in $C_{s}$ (e.g. wavenumber $k=4$ for the $32^{3}$ grid), which explains the delta-function-like shape of the p.d.f. at that specific scale. Then, there is a monotonic increase of the coefficient of variation for all scales above the 'critical' scale. The growth rate for the small scales is regular and is almost the same for all grid resolutions (as long as a proper normalization by the interval size is made).

Obviously, figure 10(a) illustrates that the LES solution is sensitive to $C_{s}$ for all scales. From a conceptual point of view, one might expect changes in $C_{s}$ to mainly affect the small scales, which govern the dissipation mechanism (see e.g. Jiménez 1999). The growth of the coefficient of variation for small scales (large $k$ ) in figure $10(a)$ further confirms this.

In figure $10(b)$, the coefficient of variation is presented for centred distributions with a decreasing variance (cf. figure $8 a$ ). We present results for the $64^{3}$ resolution. The $32^{3}$ and $48^{3}$ results (not shown) display similar trends. We observe a vertical shift between curves, associated with the different variance in the input distribution. The coefficient of variation of the small scales remains linear with $k$. A careful examination of these 

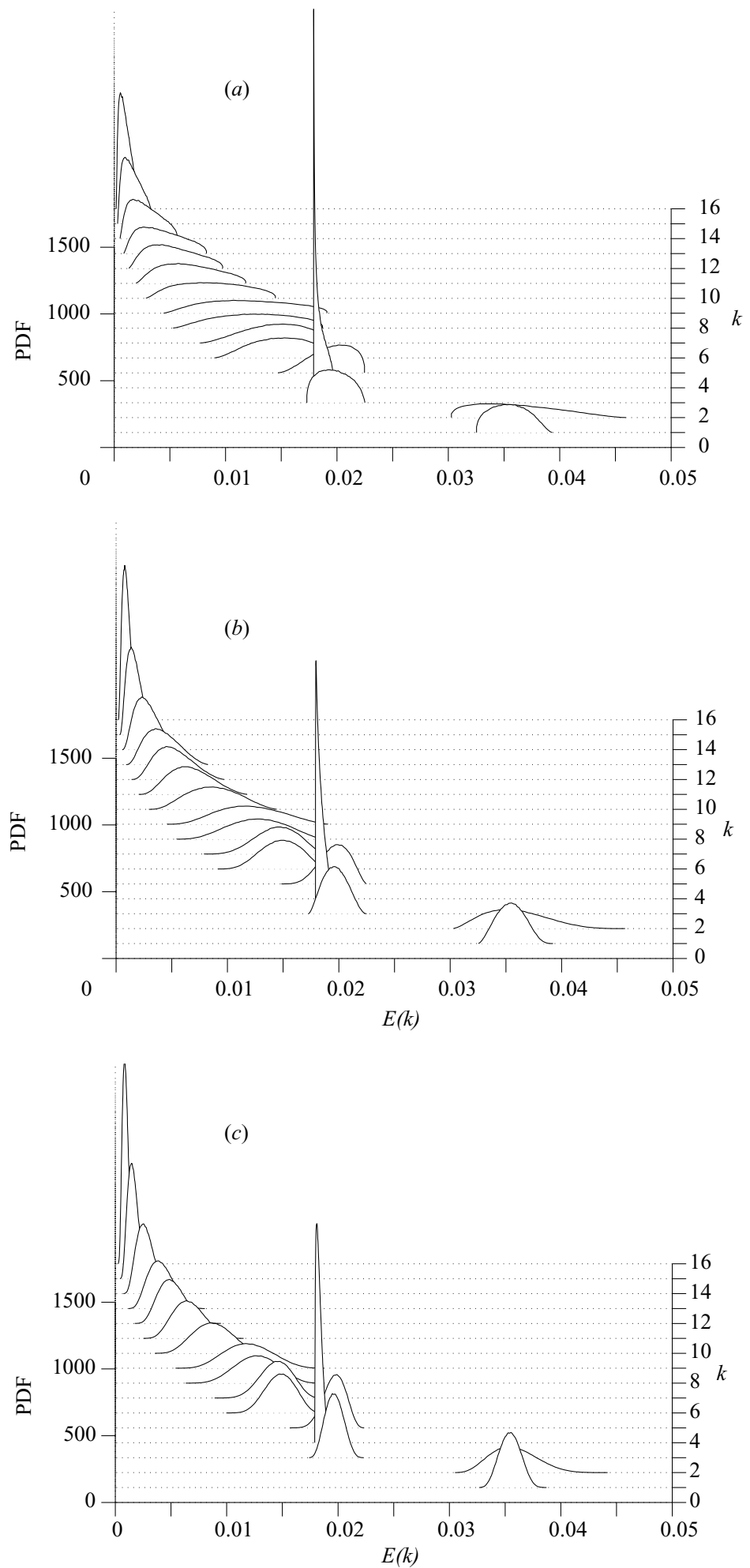

FIGURE 9. Turbulent kinetic energy p.d.f. results for $32^{3}$ grid cells with $C_{s}$ following a beta distribution $(a) B(2,2),(b) B(5,5),(c) B(10,10)$. 

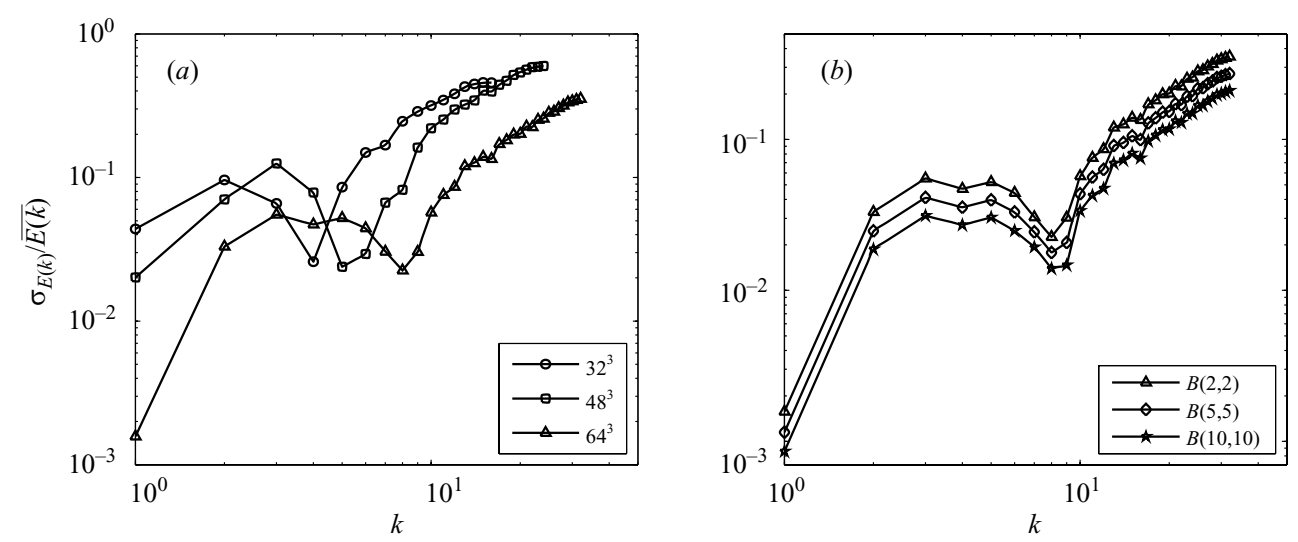

FIGURE 10. Coefficient of variation $c_{v}=\sigma_{E(k)} / \overline{E(k)}$ of the turbulent kinetic energy. (a) $C_{s}$ follows a beta distribution $B(2,2)$. The results are compared for the different grid resolutions. (b) $C_{s}$ follows centred beta distributions (cf. figure $8 a$ ) for the $64^{3}$ grid.

results indicates that its slope is directly proportional to the $C_{s}$ variance, e.g. a smaller variance in $C_{s}$ induces a smaller variance of the spectrum. The observed linear (with $k$ ) increase of the coefficient of variation for the small turbulent scales is - to the authors' knowledge - never reported in the literature, and might be related to the mechanisms governing 'Smagorinsky turbulence'.

The skewness of the spectrum may provide further useful information. We recall that positive-valued skewness indicates that the p.d.f.s are skewed to the right, i.e. the right tails are longer than the left tails. Smaller values for the turbulent kinetic energy are then more likely to be obtained. In figure 11, the skewness of the p.d.f. of the spectra is displayed versus the wavenumber. Different distributions for $C_{s}$ and different resolutions are included. For the large scales, we observe that the sign of the skewness varies considerably (cf. figure 11a). For the small scales, and for all resolutions, the growth of the skewness is regular, and linear for large values of $k$.

We now consider the effect of non-symmetric beta distributions for the coefficient $C_{s}$, which imposes in this case that $\alpha \neq \beta$. These distributions have a non-zero skewness. These distributions are represented in figure $8(b)$. The mean values $\mu_{B(\alpha, \beta)}$ of these distributions are chosen such that they are located at the optimal $C_{s}$ value (in the sense of figure 6), or at its symmetric point relative to the centre of the interval. The symmetric non-skewed distributions with $\mu_{B(\alpha, \beta)}=\frac{1}{2} C_{s} / C_{s}^{+}$are kept as reference. The first set of distributions (solid lines) share the same variance as $B(2,2)$ (i.e. $\left.\sigma_{B(\alpha, \beta)}^{2}=\sigma_{B(2,2)}^{2}\right)$. The second set (dashed lines) share the same variance as $B(10,10)$. Further, the skewness of $B\left(\alpha_{1}, \beta_{1}\right)$ has the same magnitude as the skewness of $B\left(\alpha_{3}, \beta_{3}\right)$ but with opposite sign. Similarly, the skewness of $B\left(\alpha_{2}, \beta_{2}\right)$ and $B\left(\alpha_{4}, \beta_{4}\right)$ are the same.

Figure 11(b) shows the skewness distributions of the medium and small scales for different skewed $C_{s}$ distributions (cf. figure $8 b$ ) and the $64^{3}$ case. The linear growth with $k$ is conserved for all cases. However, there is a clear vertical shift of the responses, depending on the sign of the $C_{s}$ input skewness. When the input skewness is zero, the skewness of the spectrum distribution takes values close to zero. When the input skewness is positive and the mean input value is lower than $\frac{1}{2} C_{s} / C_{s}^{+}$, the skewness of the spectrum p.d.f. is negative. When the input skewness is negative and the mean input value coincides with the optimal $C_{s}$, the resulting skewness is positive. 

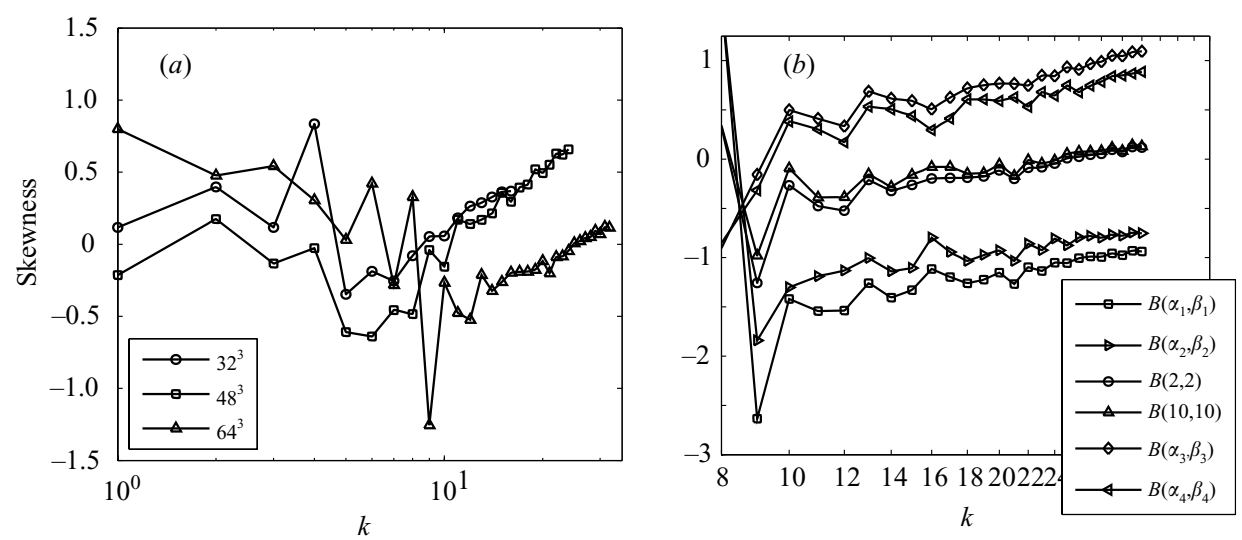

Figure 11. Skewness of the turbulent kinetic energy. (a) $C_{s}$ follows a beta distribution $B(2,2)$. The results are compared for the different grid resolutions. $(b) C_{s}$ follows skewed beta distributions (cf. figure $8 b$ ) for the $64^{3}$ grid. Approximate values for the parameters are: $\alpha_{1}=\beta_{3}=0.36, \beta_{1}=\alpha_{3}=2.38, \alpha_{2}=\beta_{4}=4.14$ and $\beta_{2}=\alpha_{4}=11.72$.

A change in variance of the input p.d.f.s is seen to play a much smaller role for the output p.d.f.s, and results appear in closely matched pairs characterized by the mean and skewness of their input distributions.

Results presented above show that the smallest resolved scales exhibit the largest relative variation to a change in $C_{s}$. This is in agreement with the general idea that the turbulent motion at small scales is more 'random' than the large-scale motion. This view yielded the proposition of the local-chaos hypothesis (McComb, Hunter \& Johnston 2001). The observed behaviour of the skewness of the p.d.f. of the smallest resolved scales corresponds to the dynamical picture that a preferentially underestimated value of $C_{s}$ will yield a preferential overestimation of the kinetic energy of these scales, related to an energy pile-up. The change in the sign of the skewness of the p.d.f. between very large and small scales can also be interpreted. An overestimation of the smallest-scale kinetic energy may result in a too high kinetic energy transfer rate from large to small scales due to triadic interactions, i.e. an accelerated forward energy cascade process, leading to an underestimation of the large-scale energy. On the other hand, an underestimation of the smallest-scale kinetic energy can yield a too small energy-transfer rate and the so-called 'bottleneck effect', i.e. an overestimation of the large-scale kinetic energy. The present gPC modelling allows a useful evaluation of these phenomena.

\section{Concluding remarks}

The results presented above show that the sensitivity of the LES solution to uncertain $C_{s}$ can be successfully investigated using a generalized Polynomial Chaos method. In order to obtain an efficient and fast converging parameterization of the space of possible solutions spanned by uncertainties, a non-intrusive decomposition of statistical moments of the LES solutions should be performed. On doing so, the lack of convergence observed in the early trials to apply the Wiener-Hermite decomposition to an instantaneous turbulent velocity field is prevented.

The pseudo-spectral representation of uncertainty offered by this method makes it possible to get insight into the sensitivity of the solution. We have shown in the present paper how response surfaces can be reconstructed and analysed via usual 
indicators such as probability density function, mean value, variance etc. The gPC approach was shown here to be able to provide an accurate statistical description (including the p.d.f.) of the space of possible LES solutions with minimum effort, since the data are approximated with spectral accuracy. It offers a systematic and mathematically grounded way to replace the usual sensitivity analysis of the solution found in most papers presenting numerical results, in which a low-dimensional finite set of computed solutions are visually compared, without quantitative analysis of the sensitivity.

The results presented above also show that the sensitivity analysis may provide a useful step in finding optimal values of some unknown parameters appearing in the mathematical model. In the present case, it has been observed that a grid-dependent value of the Smagorinsky constant, $C_{s}^{+}$, can be identified. For $C_{s}>C_{s}^{+}$, the computed energy spectra are very similar to the usual spectrum shapes observed in the viscous range of DNS spectra, showing that the subgrid-viscosity-induced dissipation is dominant at almost all scales. An interesting result is that this critical value is very close to the usual heuristic values found for $C_{s}$ in the literature. This may lead to the definition of an a priori criterion for the choice of the optimal $C_{s}$, and is an interesting subject for further research.

Finally, the present work suggests that the construction of accurate response surfaces relying on mathematically grounded approaches should be preferred to 'brute force' sampling for validation and analysis purposes.

\section{REFERENCES}

Askey, R. \& Wilson, J. 1985 Some basic hypergeometric polynomials that generalize Jacobi polynomials. Mem. Am. Math. Soc., AMS, Providence RI, vol. 319.

AsoKan, B. V. \& ZABARAS, N. $2005 a$ Using stochastic analysis to capture unstable equilibrium in natural convection. J. Comput. Phys. 208, 134-153.

AsokAn, B. V. \& ZABARAS, N. 2005b Variational multiscale stabilized fem formulations for transport equations: stochastic advection-diffusion and incompressible stochastic NavierStokes equations. J. Comput. Phys. 205, 94-133.

CAmeron, R. \& Martin, W. 1947 The orthogonal development of nonlinear functionals in series of Fourier-Hermite functionals. Ann. Math. 48, 385.

Canavan, G. 1970 Some properties of a Lagrangian Wiener-Hermite expansion. J. Fluid Mech. 41, 405-412.

Carati, D., Winckelmans, G. S. \& Jeanmart, H. 2001 On the modelling of the subgrid-scale and filtered-scale stress tensors in large-eddy simulation. J. Fluid Mech. 441, 119-138.

Chorin, A. 1974 Gaussian fields and random flow. J. Fluid Mech. 85, 325-347.

Crow, S. \& Canavan, G. 1970 Relationship between a wiener-hermite expansion and an energy cascade. J. Fluid Mech. 41, 387-403.

Deardoff, J. W. 1970 A numerical study of three-dimensional turbulent channel flow at large Reynolds numbers. J. Fluid Mech. 41, 453-480.

Deodatis, G. 1991 Weighted integral method. I: stochastic stiffness matrix. J. Engng Mech. 117 (8), $1851-1864$.

Deodatis, G. \& Shinozuka, M. 1991 Weighted integral method. II: response variability and reliability. J. Engng Mech. 117 (8), 1865-1877.

Fishman, G. 1996 Monte Carlo: Concepts, Algorithms, and Applications. Springer.

Frauenfelder, P., Schwab, C. \& Todor, R. 2005 Finite elements for elliptic problems with stochastic coefficients. Comput. Meth. Appl. Mech. Engng 193, 205-228.

FRISCH, U. 1995 Turbulence. Cambridge University Press.

Fureby, C. \& TABor, G. 1997 Mathematical and physical constraints on large-eddy simulations. Theor. Comput. Fluid Dyn. 9, 85-102.

Germano, M. 1992 Turbulence: the filtering approach. J. Fluid Mech. 238, 325-336. 
Germano, M., Piomelli, U., Moin, P. \& Cabot, W. H. 1991 A dynamic subgrid-scale eddy viscosity model. Phys. Fluids A 3, 1760-1765.

GeurTs, B. J. \& FröHLICH, J. 2002 A framework for predicting accuracy limitations in Large Eddy Simulations. Phys. Fluids 14, L41-L44.

GHANEM, R. 1999 Ingredients for a general purpose stochastic finite element formulation. Comput. Meth. Appl. Mech. Engng 168, 19-34.

Ghanem, R. \& Brzakala, W. 1996 Stochastic finite-element analysis of soil layers with random interface. ASCE J. Engng Mech. 122, 361-369.

GHANEM, R. \& Dham, S. 1998 Stochastic finite element analysis for multiphase flow in heterogeneous porous media. Transp. Porous Media 32, 239-262.

Ghanem, R. \& HaYeK, B. 2002 Probabilistic modeling of flow over rough terrain. Trans. ASME: J. Fluids Engng 124, 42-50.

Ghanem, R. \& Red-Horse, J. 1999 Propagation of uncertainty in complex physical systems using a stochastic finite elements approach. Physica D 133, 137-144.

Ghanem, R. \& Spanos, P. 1991 Stochastic Finite Elements: a Spectral Approach. Springer.

Ghosal, S. 1996 An analysis of numerical errors in large-eddy simulations of turbulence. J. Comput. Phys. 125, 187-206.

Ghosal, S. 1999 Mathematical and physical constraints on large-eddy simulation of turbulence. AIAA J. 37, 425-433.

Hien, T. \& Kleiber, M. 1997 Stochastic finite element modelling in linear transient heat transfer. Comput. Meth. Appl. Mech. Engng 144, 111-124.

Hien, T. \& Kleiber, M. 1998 On solving nonlinear transient heat transfer problems with random parameters. Comput. Meth. Appl. Mech. Engng 151, 287-299.

Hou, T. Y., Luo, W., RozovskiI, B. \& Zhou, H.-M. 2006 Wiener chaos expansions and numerical solutions of randomly forced equations of fluid mechanics. J. Comput. Phys. 216, 687-706.

Hughes, T. J. R., Mazzei, L. \& Oberai, A. A. 2001 The multiscale formulation of large eddy simulation: Decay of homogeneous isotropic turbulence. Phys. Fluids 13, 505-512.

JimÉneZ, J. 1999 On eddy-viscosity sub-grid models. In Direct and Large-Eddy Simulation III (ed. P. R. Voke, N. D. Sandham \& L. Kleiser), pp. 75-86.

JimÉNEZ, J. \& Moser, R. D. 2000 Large-eddy simulations: where are we and what can we expect? AIAA J. 38, 605-612.

KeESE, A. 2005 Numerical solution of systems with stochastic uncertainties: a general purpose framework for stochastic finite elements. PhD thesis, Technische Universitat Braunschweig, Mechanik-Zentrum.

Kleiber, M. \& Hien, T. 1992 The Stochastic Finite Element Method. John Wiley \& Sons.

KNIO, O. \& LE MAÎTRE, O. 2006 Uncertainty propagation in CFD using polynomial chaos decomposition. Fluid Dyn. Res. 38 (9), 616-640.

Koekoek, R. \& Swarttouw, R. 1998 The Askey-scheme of hypergeometric orthogonal polynomials and its q-analogue. Tech. Rep. 98-17. Department of Technical Mathematics and Informatics, Delft University of Technology.

Le Maitre, O., Knio, O., Najm, H. \& Ghanem, R. 2001 A stochastic projection method for fluid flow. i. basic formulation. J. Comput. Phys. 173, 481-511.

Le Maitre, O., Najm, H., Ghanem, R. \& Knio, O. 2004 Multi-resolution analysis of wiener-type uncertainty propagation schemes. J. Comput. Phys. 197, 502-531.

Le Maitre, O., Reagan, M., Najm, H., Ghanem, R. \& Knio, O. 2002 A stochastic projection method for fluid flow. ii. random process. J. Comput. Phys. 181, 9-44.

LI, R. \& Ghanem, R. 1998 Adaptive polynomial chaos expansions applied to statistics of extremes in nonlinear random vibration. Prob. Engng Mech. 13 (2), 125-136.

LiLLY, D. K. 1966 On the application of the eddy-viscosity concept in the inertial sub-range of turbulence. Tech. Rep. 123. NCAR.

LiLly, D. K. 1967 The representation of small-scale turbulence in numerical simulation experiments. In Proceedings of IBM Scientific Computing Symposium on Environmental Siences. IBM Data Processing Division, White Plains, New York.

LiU, P.-L. \& Der KiUREghian, A. 1991 Finite element reliability of geometrically nonlinear uncertain structures. J. Engng Mech. 117, 1806-1825. 
LuCor, D. 2004 Generalized Polynomial Chaos: applications to random oscillators and flowstructure interactions. $\mathrm{PhD}$ thesis, Brown University.

LuCor, D. \& KARNiadakis, G. 2004a Adaptive generalized polynomial chaos for nonlinear random oscillators. SIAM J. Sci. Comput. 26 (2), 720-735.

Lucor, D. \& Karniadakis, G. $2004 b$ Noisy Inflows Cause a Shedding-Mode Switching in Flow past an Oscillating Cylinder. Phys. Rev. Lett. 92, 154501-1; 154501-4.

McComb, W. D., Hunter, A. \& Johnston, C. 2001 Conditional mode elimination and the subgrid modeling problem for isotropic turbulence. Phys. Fluids 13, 2030-2044.

McMillan, O. J. \& Ferziger, J. H. 1979 Direct testing of subgrid-scale models. AIAA J. 17, $1340-1346$.

Meecham, W. \& Jeng, D. 1968 Use of the wiener-hermite expansion for nearly normal turbulence. J. Fluid Mech. 32, 225-249.

Meneveau, C. 1994 Statistics of turbulence subgrid-scale stresses: Necessary conditions and experimental tests. Phys. Fluids 6, 815-833.

Meneveau, C. \& Lund, T. S. 1997 The dynamic Smagorinsky model and scale-dependent coefficients in the viscous range of turbulence. Phys. Fluids 9, 3932-3934.

Meyers, J., Geurts, B. J. \& Baelmans, M. 2003 Database-analysis of errors in large-eddy simulation. Phys. Fluids 15, 2740-2755.

Meyers, J., Geurts, B. J. \& Baelmans, M. 2005 Optimality of the dynamic procedure for large-eddy simulations. Phys. Fluids 17, 045108.

Meyers, J. \& Sagaut, P. 2006 On the model coefficients for the standard and the variational multi-scale Smagorinsky model. J. Fluid Mech. 569, 287-319.

Meyers, J., Sagaut, P. \& Geurts, B. J. 2006 Optimal model parameters for multi-objective largeeddy simulations. Phys. Fluids 18, 095103.

Nicoud, F. \& Ducros, F. 1999 Subgrid-scale stress modelling based on the square of the velocity gradient tensor. Flow Turbul. Combust. 62, 183-200.

Ogura, H. 1972 Orthogonal functionals of the Poisson process. IEEE Trans. Info. Theory IT-18, 473-481.

OrszaG, S. \& BissonnetTe, L. 1967 Dynamical properties of truncated Wiener-Hermite expansions. Phys. Fluids 10, 2603-2613.

Pope, S. B. 2000 Turbulent Flows. Cambridge University Press.

Porté-Agel, F., Meneveau, C. \& Parlange, M. B. 2000 A scale-dependent dynamic model for large-eddy simulation: application to a neutral atmosoheric boundary layer. J. Fluid Mech. 415, 261-284.

SAGaut, P. 2006 Large Eddy Simulations for Incompressible flows, 3rd edn. Springer.

Schoutens, W. 1999 Stochastic processes in the Askey scheme. PhD thesis, K.U. Leuven.

Shinozuka, M. \& Deodatis, G. 1988 Response variability of stochastic finite element systems. J. Engng Mech. 114, 499-519.

Smagorinsky, J. 1963 General circulation experiments with the primitive equations: I. The basic experiment. Mon. Weath. Rev. 91, 99-165.

Spanos, P. \& Ghanem, R. 1989 Stochastic finite element expansion for random media. ASCE J. Engng Mech. 115, 1035-1053.

Tatang, M., Pan, W., Prinn, R. \& McRae, G. 1997 An efficient method for parametric uncertainty analysis of numerical geophysical models. J. Geophys. Res. 102, 21925-21932.

VANMARCKe, E. \& GrigoriU, M. 1983 Stochastic finite element analysis of simple beams. J. Engng Mech. 109, 1203-1214.

Völker, S., Moser, R. D. \& Venugopal, P. 2002 Optimal large eddy simulation of turbulent channel flow based on direct numerical simulation data. Phys. Fluids 14, 3675-3691.

VReman, A. W. 2004 An eddy-viscosity subgrid-scale model for turbulent shear flow: Algebraic theory and applications. Phys. Fluids 16, 3670-3681.

Vreman, B., Geurts, B. \& Kuerten, H. 1996 Comparison of numerical schemes in large-eddy simulations of the temporal mixing layer. Intl J. Numer. Meth. Fluids 22, 297-311.

Vreman, B., Geurts, B. J. \& Kuerten, H. 1994 Realizability conditions for the turbulent stress tensor in large-eddy simulation. J. Fluid Mech. 278, 351-362.

VoKe, P. R. 1996 Subgrid-scale modelling at low mesh Reynolds number. Theor. Comput. Fluid Dyn. 8, 131-143. 
Wan, X. \& Karniadakis, G. 2005 An adaptive multi-element generalized polynomial chaos method for stochastic differential equations. J. Comput. Phys. 209, 617-642.

WAN, X. \& Karniadakis, G. 2006 Stochastic heat transfer enhancement in a grooved channel. J. Fluid Mech. 565, 255-278.

Webster, M., Tatang, M. \& McRae, G. 1996 Application of the probabilistic collocation method for an uncertainty analysis of a simple ocean model. MIT Joint Program on the Science and Policy of Global Change Reports Series 4. MIT.

Wiener, N. 1938 The homogeneous chaos. Am. J. Maths 60, 897-936.

XiU, D. \& Karniadakis, G. 2002 The Wiener-Askey polynomial chaos for stochastic differential equations. SIAM J. Sci. Comput. 24, 619-644.

XiU, D. \& Karniadakis, G. 2003a Modeling uncertainty in flow simulations via generalized polynomial chaos. J. Comput. Phys. 187, 137-167.

XiU, D. \& Karniadakis, G. 2003b A new stochastic approach to transient heat conduction modeling with uncertainty. Intl J. Heat Mass Transfer 46, 4681-4693.

ZHANG, D. \& Lu, Z. 2004 An efficient, high-order perturbation approach for flow in random porous media via karhunen-loeve and polynomial expansions. J. Comput. Phys. 194, 773-794.

Zhu, W., ReN, Y. \& WU, W. 1992 Stochastic FEM based on local average of random vector fields. J. Engng Mech. 118, 496-511.

ZHU, W. \& WU, W. 1991 A stochastic finite element method for real eigenvalue problems. Prob. Engng Mech. 6, 228-232. 